\title{
The geography of open fractures of the lower limb: Access to trauma care
}

\author{
Georgia Hawthorne, Anand Pillai and Mohamad Alqubaisi* \\ University Hospital of South Manchester NHS Foundation Trust, UK
}

\begin{abstract}
The aim of this paper is to develop an understanding of and document the geographical distribution of patients who suffered open fractures to the lower extremity between May 2013 and May 2015 and were treated at University Hospital of South Manchester (UHSM). To be able to recognise and explore any patterns in demographics and distances from home to hospital. This information will be used to review access to trauma care. Guidance to reduce trauma services in the region will be reviewed and any implications explored. On average the home to hospital distance was above the national average and would increase further if reconfiguration recommendations were followed. The reason for these higher values will be explored and the demographics of the two data sets compared to others in the literature. The data of orthoplastic patients and those only requiring orthopaedic care will be compared.
\end{abstract}

\section{Introduction}

Although major traumas are uncommon, representing only $0.1 \%$ of cases treated in NHS A\&E departments the complexity of their nature requires treatment at a specialised major trauma centre [1]. It is not possible for all hospitals to be equipped to deal with the severe and specialist injuries often acquired. Therefore, there is a need for a more specialist network of centres to be able to deal with these cases. As of April 2012, 22 major trauma networks were developed each revolving around a major trauma centre, there are 26 centres in the UK, 2 of these are collaborative centres made up of more than one hospital. UHSM is part of the Greater Manchester collaborative major trauma centre with 2 other hospitals; Manchester Royal infirmary (MRI) and Salford Royal NHS trust [2]. There have been major advances in the management of open fractures of the lower extremity in the UK over the previous decade following the initiation of structured networks. Development of recognised centres to deal with trauma has allowed collaborative work between specialties in one site and at an earlier point in the patient journey. It is a requirement of major trauma centres to be able to treat lower extremity open fractures on site, requiring both orthopaedic and plastic surgical involvement $[3,4]$.

Previously patients involved in major trauma were taken to the nearest hospital, evidence-based approaches found a clear advantage in presentation to a major trauma centre. There have also been huge improvements to pre-hospital care received by patients [3]. Trauma care in the UK for many years did not match international standards, despite numerous peer reviews and reports from TARN leading to the first National Clinical Director of Trauma being appointed in 2008. Concerns of trauma care shortfalls in the UK were raised further in 2010 via publication of a national audit office report, comments about failure in restructuring services lead to major trauma centres going live by April 2012 [5]. Recent reviews have suggested changes to the structure of the greater Manchester collaborative to deliver services from only one of the hospitals involved [1]. With trauma network structures so new there is great benefit in reviewing the geographical distribution of whom these services reach and any possible implications of restructuring current services.

\section{Literature review}

Search criteria and metrics: I used reputable online sources to search for relevant papers such as Medline using search criteria of lower limb open fractures and access to trauma. I limited my literature selections to UK and the last 5 years mainly. For some data sets there was advantage in comparing changes over time. I used reliable sources such as NHS trauma network information and department of transport data for comparison.

\section{Changing demographics of major trauma}

Despite major trauma being rare, injuries are often severe resulting in major morbidity and mortality, accounting for the major cause of death in those under 40 [5]. Population distribution changes are leading to changes in the demographics of major trauma; the ageing population has led to shifting causes. Previously the main cause was high-energy transfer events such as RTAs affecting mainly younger patients; more injuries now result from falls from low height, which are common in the elderly population. According to the TARN database in 1990 RTAs accounted for $59.1 \%$ of major trauma injuries with $39.3 \%$ in under 24-year olds. By 2013 the shifting cause of major trauma lead to a change in the proportions of populations affected, the mean age increased to 53.8 years and the age group most commonly affected was now 25-50 years, the 75+ age group was a very close second [6]. As the age of the population continues to rise so will the age of patients suffering major trauma, meaning a more diverse care package is needed for patients such as including geriatricians and neurologists to deal with head injuries commonly seen in fall patients. Changes in the mechanism of injury from RTAs to small height falls (less than $2 \mathrm{~m}$ ), such as falling down stairs could affect the distribution of where trauma

${ }^{*}$ Correspondence to: Mohamad Alqubaisi, University Hospital of South Manchester NHS Foundation Trust, UK, E-mail: mohamad.alqubaisi@mft.nhs.uk

Received: September 24, 2018; Accepted: October 13, 2018; Published: October 17,2018 
occurs. Moving away from major roads to residential properties making the proximity of major trauma centres to residential areas increasingly important [6].

\section{Access to emergency and trauma care}

Accessibility to health care for many indicatives of funding, with A\&E department closures attracting high levels of public interest. A review into emergency care in 2012 recommended reconfigurations into how emergency care is delivered, suggesting primary care services used more often to elevate the burden of inappropriate presentation at A\&E departments [7]. There are currently 200 sites that provide major emergency care and only 22 networks over 26 sites providing major trauma care in the UK $[2,7]$. There has been $8 \%$ net reduction in site numbers that provide emergency care since 2001-2014 [7]. If current recommendations to close 2 of the 3 sites in the Greater Manchester collaborative are followed this would lead to a $7 \%$ reduction to trauma sites within only 2 years, losing essential trauma services. Although uncommon, the impact of individual closures can be profound. In parts leading to increases in distance travelled by patients to $\mathrm{A} \& \mathrm{E}$ departments of up to $5 \mathrm{~km} \mathrm{[7].}$

During 2011/2012 patients travelled an average of $7.2 \mathrm{~km}$ to attend an A\&E department, this increased to $8.7 \mathrm{~km}$ for an emergency admission, $80 \%$ of attendances were by people living within $12 \mathrm{~km}$ of the site. Distances vary by local authority being as little as $2.5 \mathrm{~km}$ average to attend $\mathrm{A} \& \mathrm{E}$ in some areas, general trends showed distance travelled was directly proportional to the scarceness of population in the area according to population density data [7].

Data for all emergency admissions was compared to emergency admissions due to stoke and trauma for 2011/2012 with trauma representing $10 \%$ of admissions, with a greater proportion of these patients travelling $30+\mathrm{km}$ for treatment. RTA data showed $80 \%$ involved needed trauma care, suggesting the reason trauma patients travel further could be due to the mechanism of injury, namely RTA occurring away from homes and that the distance from trauma occurrence to trauma care would have been less. RTA patients' houses were on average $12.3 \mathrm{~km}$ away from the A\&E department they attended, much larger than other causes of admission [7]. With changing demographics leading to a change in causes of trauma the distribution of injury sites may also change making house to hospital distances increasingly important [6].

It was also considered that transfer from another hospital to trauma centre might have been accountable for some of the increase in difference, however average distance between house and trauma centre when a patient presented there was $10.8 \mathrm{~km}$ which is not significantly different to average distance travelled by trauma patients to other hospitals at $9.95 \mathrm{~km}$ average [7]. This would suggest that trauma patients received care further from their homes because there are less trauma centres than $\mathrm{A} \& \mathrm{E}$ departments due to the specialist level of care needed, this could worsen if recommendations to close trauma centres within the Greater Manchester Collaborative are followed [1]. There were 200 sites proving A\&E services to 5.2 million patients for the time period studied, trauma represented $10 \%$ of these patients treated at $15 \%$ of the amount of sites as there are 30 hospitals involved in the major trauma network, this could lead one to conclude that major trauma care is overrepresented in comparison to other care types, however reducing the trauma centres to 20 would hugely increase the distances travelled from patients homes to hospital [7]. The average UK commuter travels $14 \mathrm{~km}$ to work each day, with males traveling an average of $17 \mathrm{~km}$, which is $67 \%$ further than women at $10.1 \mathrm{~km}$; this gives an insight into how far people are willing to travel for normal daily activities [8].

\section{Staffing of major trauma centres}

Major trauma services are essential components of care at major trauma centres, comprised of general surgeons in most trusts. Shortfalls have been identified in the transfer from initial assessment to surgery and treatment, suggesting a need for a more rigid structure of staffing in delivering care, with a definitive management role for trauma scenarios [10]. Out of 22 centres studied only 13 currently have a trauma service in place, with many failings to even consider such an essential staffing network. Since major trauma centres went live in April 2012 the workload of all specialities involved has increased, plastic surgeons have seen a 7 -fold increase in workload [10]. Many have suggested the need for a recognised sub specialty for dealing with trauma cases, helping to explain the gap between UK and global outcomes [6]. The initiation of a trauma network helps bridge the gap but is not providing enough structure to allow the UK to excel in treating patients involved in major trauma. Understanding the roles of different specialties in patient care would help in structuring service designs and improving training to incorporate such aspects.

\section{Management protocol}

Protocol devised by The British Orthopaedic association and The British Association of Reconstructive and Aesthetic Surgeons outlines management standards for open fractures of the lower limb and are based on best practice. The guidance (BOAST4) requires the combination of orthopaedic and plastic surgical specialists at the same site, which is not always a reality. With an orthoplastic approach, reconstruction has been as successful as $98.5[3,11]$. BOAST4 criteria specifies a need to start IV infusion of antibiotics within 3 hours of the accident when possible, in one study this occurred in $97 \%$ of patients who presented to a trauma centre and only $24 \%$ of patients presenting to A\&E [3-12]. This shows the advantages of presenting to a trauma centre and the importance of geographical proximity in infection prophylaxis.

BOAST4 recommends debridement as soon as possible by specialists and at most within 24 hours, post April 2012 this has been achieved in $94 \%$ of cases compared to only $34 \%$ prior to the establishment of the trauma network [3-12]. A structured network approach and enforced protocol based on best evidence allows for improved results in trauma cases. The 6 hour rule in regards to debridement has shown no improvements in infection rates and is no longer accepted in the literature, one study assisted by the institution of inflammation and repair in Manchester University found deep infection rates greater when debridement was done more than 6 hours after injury in one data set but no statistical differences post 6 hour rule on meta-analysis of 7 data sets [12].

NHS protocol recommends a minimum of 12 month follow up for patients who have undergone major trauma, for care continuity if patients were to have to travel long distances to see the same practitioner's mental recovery and adaptation to normal life following potentially life altering injuries could be impeded $[2,13]$. In the case of RTA patients social support and regaining confidence is vital, a process that may be hindered if the patients are unable to attend appointments unaided [13].

\section{Trauma network reviews and recommendations}

According to a national peer review report in 2015, the structure of the major trauma network has led to improved outcomes and decreased mortality. 
The Greater Manchester trauma network scored $89 \%$ on network governance measures which is second highest, rehabilitation scored were also high but definitive care amongst the lowest of all networks with 50\% [14]. This low score may result from distinctly different practices across the 3 sites. There was above average compliance to BOAST4 guidelines and strong clinical leadership, with improvements to TARN databases recommended. Concerns were raised in the lack of overall coordinated care for patients with multiple injuries and lack of daily review with all specialties and therapy teams, it was suggested that 1 hospital become the major receiving site allowing for a more cohesive service [14,15]. An NHS report found that 3 centres is not suitable for running a trauma network long term and that all 3 must be able to meet high national standards [1]. Care delivery from all 3, only 2 and finally just 1 site were considered by an expert panel using 5 main concepts; lives saved and life quality of survivors, patient numbers, injury types, geographical distribution of injuries and clinical facilities and their distribution. The main recommendations were for all sites to move to 24/7 major trauma consultant A\&E cover from 2015 and a reduction to only one major trauma site within 2 years [1]. Despite considering many aspects the distance from patients' homes to hospital had been overlooked, this could impede long-term recovery due to attending outpatient appointments or receiving inpatient visits from family and friends. The changing demographics of trauma injuries should be considered when making long-term decisions.

When considering which sites to close in the Greater Manchester Collaborative facilities supplied by each trust along with performance should be considered. Based on CQC reports the best performing trust was Salford Royal, which achieved outstanding. However, when considering the components required for trauma cases such as surgery and intensive and critical services the results are more equal, with MRI the only hospital scoring good in both areas [16]. Facilities at each site have a major weighting in decisions made, if a trust is unable to facilitate the complex needs of patients on a single site it should not be considered capable of such a role. Current guidance states that trauma centres must be able to treat open fractures of the lower limb, and that this requires both plastic and orthopaedic surgical specialties on site $[3,4]$. UHSM is the only site in the Greater Manchester Collaborative that offers both specialties. BOAST4 guidance remarks on the importance of revascularisation and surgical repair of and vascular injuries as quickly as possible, again UHSM is the only site able to facilitate this onsite, with specialist vascular surgeons [10,17-19].

Guidelines have recommended a move away from primitive practices of rushed debridement and fixation at a non-specialist hospital then movement to a trauma centre, moving patients from Salford to Wythenshawe for plastic surgical specialist intervention may reverse advances in protocol and best evidence recommendations [20].

\section{Audit aims and standards}

The aims of this audit were to review and contribute to the limited literature exploring access to trauma care in the UK. Studies considering access to emergency care are easily accessible, but few studies look only at access to trauma care. I reviewed the distributions of patients' home addresses as a measure of areas served by UHSM, producing a map to illustrate this. Moving patients between centres and from other hospitals to more specialist trauma centres presents time constraints and delays in initiation of treatment specifically antibiotic administration and debridement, which can influence recovery [11]. I wanted to assess if following recommendations to reduce the Greater Manchester collaborative to one site would benefit or hinder patients $[1,2]$. Patients requiring orthoplastic involvement data were compared to patients needing only orthopaedic input, to help establish the full extent of disruption if the Greater Manchester Collaborative was changed to Salford Royal only, which would be unable to care for orthoplastic patients onsite.

\section{Methodology}

This was a retrospective study of patients who had been treated at UHSM for open fractures of the lower limb. Inclusion criteria included patients treated from May 2013 to May 2015, the patients must have undergone surgical repair by orthopaedics. There were no other selection criteria in regard to presentation or extent of injuries. A second data set also had to meet the criteria of plastic surgical involvement. The data set comprised initially of 30 patients in each cohort. Some Data was collected from another study being carried out, and the patients' details provided by a junior doctor carrying out an audit. I then used the Sunquest ICE system to confirm the patients' details and find their postcodes. PACS was used to find more patients that could be included in the study by searching all patients in those dates that had undergone multiple $\mathrm{x}$-rays of the lower extremity and then manually filtered for relevance and inclusion criteria.

On analysis 3 patients were removed due to being outliers, which would have lead to distortion of further analysis. This left a sample size of 30 orthoplastic patients and 27 orthopaedic patients.

I used satellite data to map postcodes and marked the centre of the postcode corresponding to the patient on the map, the map was produced using Google maps. I also marked on the map UHSM, MRI and Salford royal; the hospitals that form the Greater Manchester Collaborative. For distances of travel straight line distances were used that are calculated by Pythagoras theorem, these measurements were recorded to the nearest $0.1 \mathrm{~km}$.

I then further analysed the data by exploring the following variables:

- Distance to Wythenshawe hospital

- Age of the patients

- Patients gender

- Age and gender were compared for demographic insight

- Distance to Salford Royal

When analysing the above variables, their implications on distances lived away from UHSM were also analysed. I chose these criteria for further analysis based on findings in other studies. I compared age and gender to test the theory of injuries being common in young males [6]. I also calculated distances from patients' homes to Salford Royal (the proposed single site) to assess how or if patients' journey lengths would be altered.

\section{Limitations}

The definitive aims of this project were to find out how far patients travelled to access trauma care, this could have been improved upon by mapping the site where the injury occurred and considering the distance of travel to receive trauma care rather than mapping patients home addresses. However, by mapping home addresses it gives an insight into recovery obstacles if patients have to travel far for specialised outpatient clinics if their mobility is impeded.

Calculating distances by straight line method may not be a true representation of the distances travelled, however using fastest road 
routes could also be unrepresentative as patients may not take the routes used or may rely on public transport. Further analysis into journey types and use of journey times could help.

The size of the data set was relatively small which may not fairly represent the population of patients that suffered open fractures to the lower limb and were treated at UHSM.

\section{Results and analysis}

Distances travelled to UHSM: Figure 1 is a map of the UK showing the patients home locations and the hospital in the Greater Manchester Collaborative.

When analysing the data there were 3 points that lay more than more than 1.5 times the interquartile range above the $3^{\text {rd }}$ quartile of the data set, these were distances of $343.9 \mathrm{~km}, 93.9 \mathrm{~km}$ and $79.5 \mathrm{~km}$. I was going to include the outliers in the statistical analysis as they made up $5 \%$ of the data set; however, including them would have skewed further results such as analysis of how distances changed with age groups. Therefor these outliers were removed; they are represented on figure 1 as orange points.

When outliers were removed the range of distances travelled by all patients was $35.3 \mathrm{~km}$ the average was $14.4 \mathrm{~km}$. Table 1 shows the distributions of distances travelled by the patients. Orthopaedic patients average distance was $14.4 \mathrm{~km}$ with a range of $35.1 \mathrm{~km}$. Orthoplastic patients' distances also had an average of $14.4 \mathrm{~km}$ with a slightly larger range of $35.2 \mathrm{~km}$. The data spread shows that more orthoplastic patients travelled further distances with 50\% travelling 15 $\mathrm{km}$ or more, compared to $44 \%$ of orthopaedic patients.
Figure 2 is a zoomed in map of the same region showing the distribution of the 57 data points more clearly. The outliers are not included.

\section{Age of patients}

The age of patients at the time of their injury was analysed and tested for trends in distances. Patients' ages ranged from 17 to 98 years, giving a range of ages of 72 years. With the average age of patients being 50.2 years. Figure 3 shows the proportion of patients in each age category for both data sets (Figures 3 and 4).

The orthopaedic patients had an average age of 43.3 and a range of 71 , there were less patients' aged 70+. The orthoplastic patients had an average age of 52.6 with a range of 76 years, $40 \%$ of the patients in the orthoplastic group were $70+$.

Distances travelled with age between the groups can be seen below in figure 5. In the orthoplastic group the youngest patients travelled the furthest.

\section{Gender of patients}

Of the 57 patients in this study 38 were male and 19 were female. With males representing $67 \%$ of all patients in the study. In the Table 1. Showing the amount categorised distances travelled by orthopaedic patients

\begin{tabular}{|c|c|c|}
\hline $\begin{array}{c}\text { Distance from home to } \\
\text { UHSM }\end{array}$ & Orthopaedic patients & Orthoplastic patients \\
\hline $0-5 \mathrm{~km}$ & 5 & 1 \\
\hline $5-10 \mathrm{~km}$ & 8 & 9 \\
\hline $10-15 \mathrm{~km}$ & 2 & 5 \\
\hline $15-20 \mathrm{~km}$ & 5 & 10 \\
\hline $20+\mathrm{km}$ & 7 & 5 \\
\hline
\end{tabular}

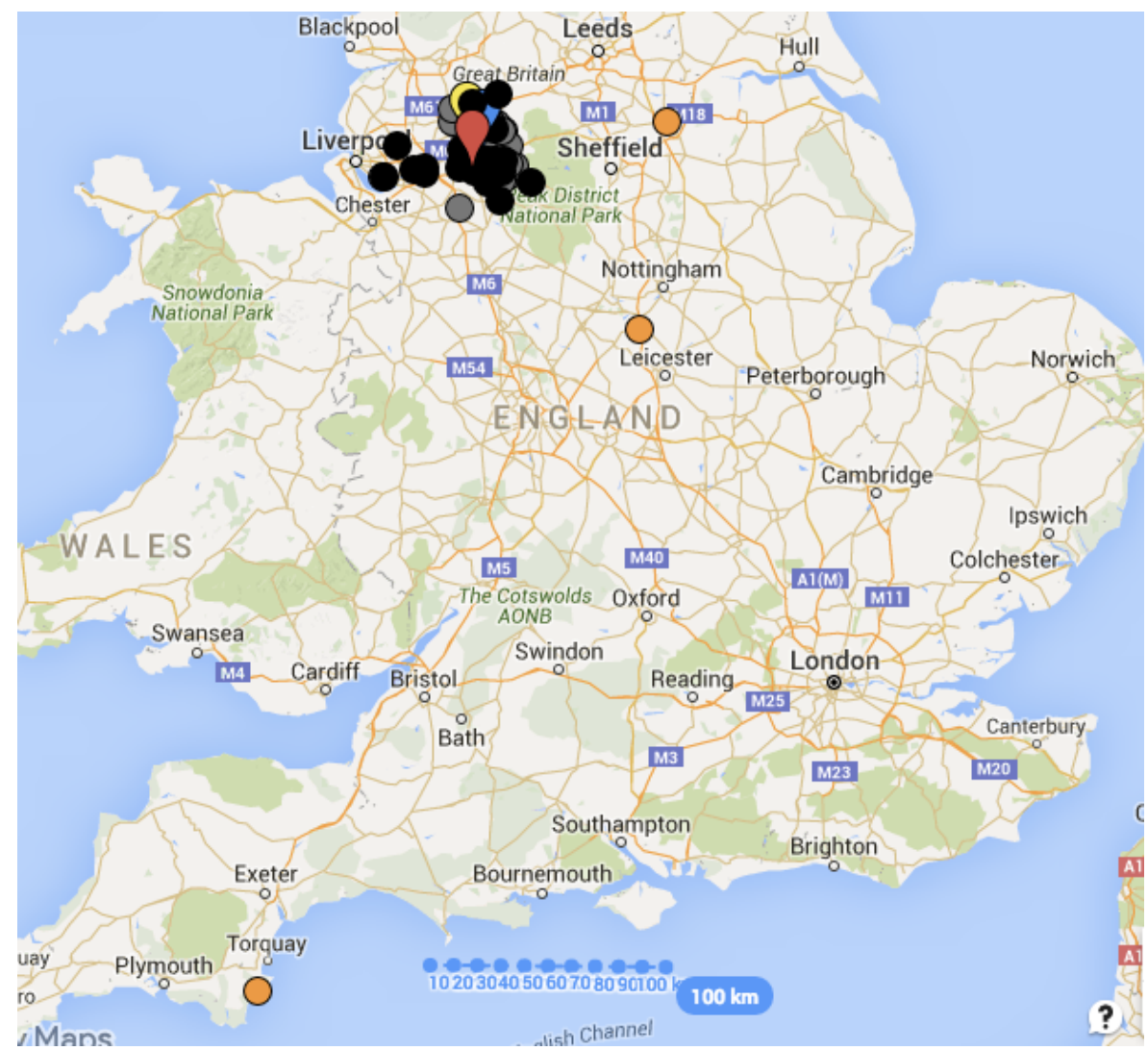

Figure 1. UK map showing distributions of patients' home addresses. Hospitals in the Greater Manchester collaborative are tear shaped icons and outliers appear orange. Orthoplastic patients shown in grey, black is orthopaedic patients 


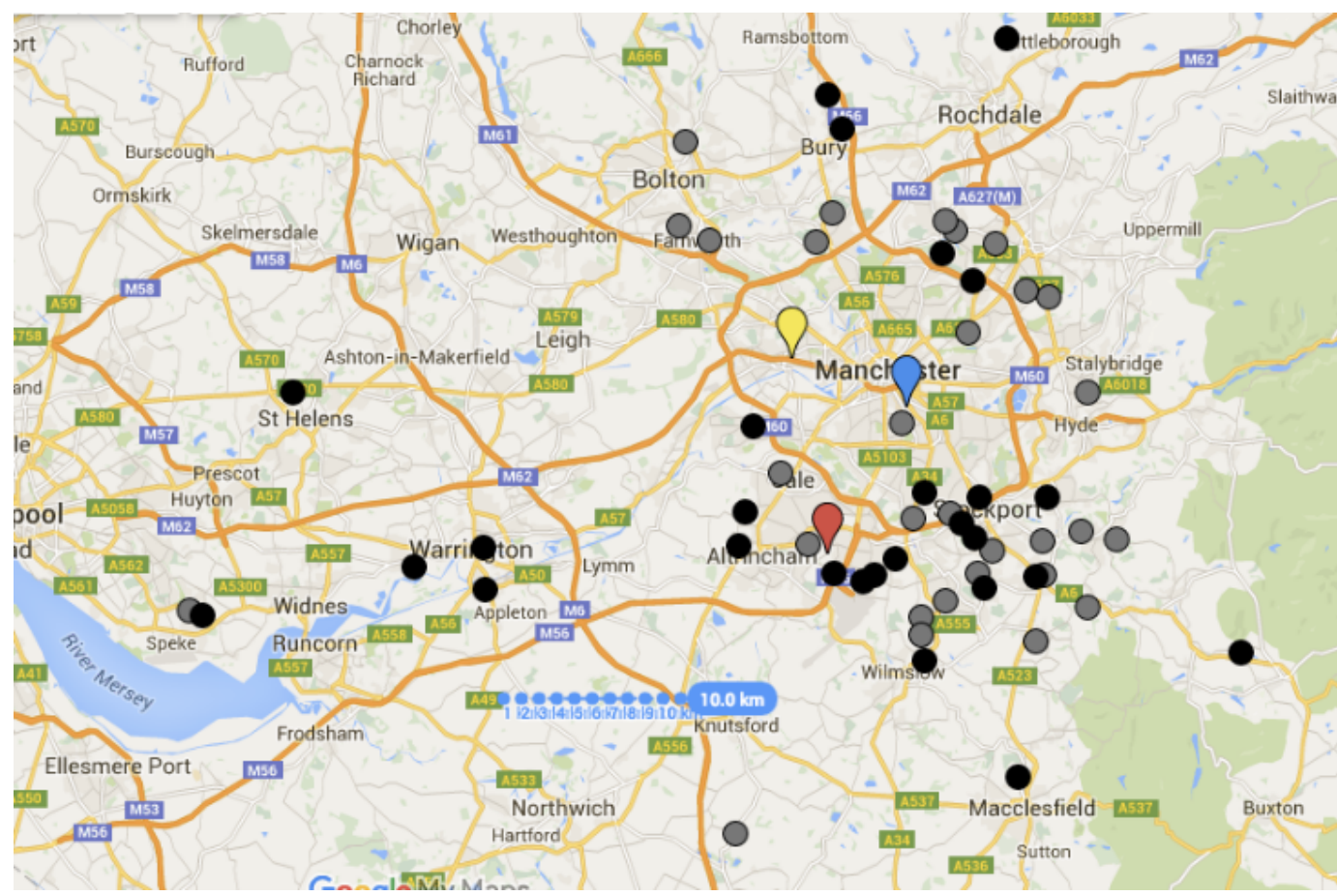

Figure 2. Zoomed map of northwest showing distributions of patients' home addresses. Orthoplastic patients shown in grey, black is orthopaedic patients. Hospitals in the Greater Manchester collaborative are tear shaped icons

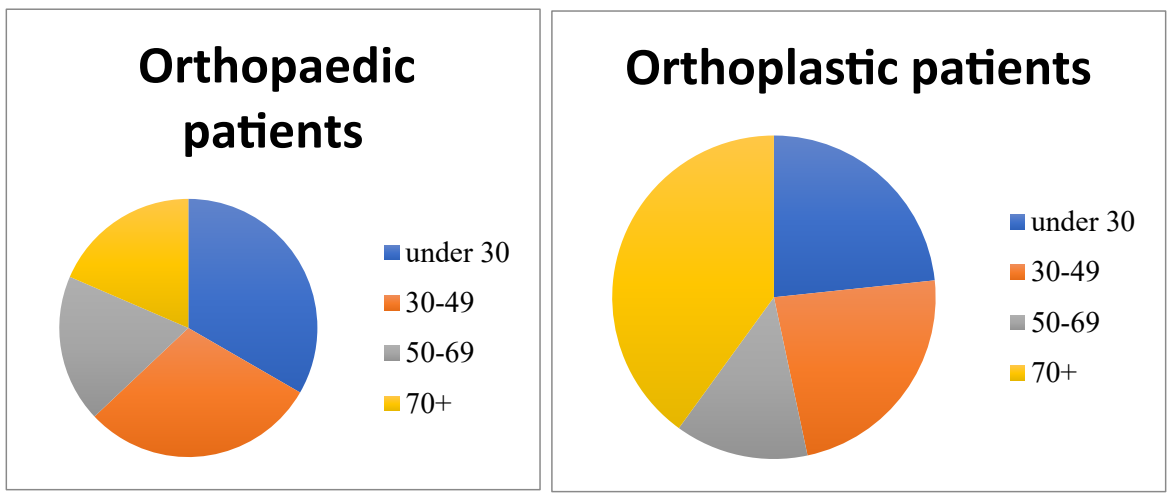

Figures 3 and 4. Pie charts showing proportions of patients at each age

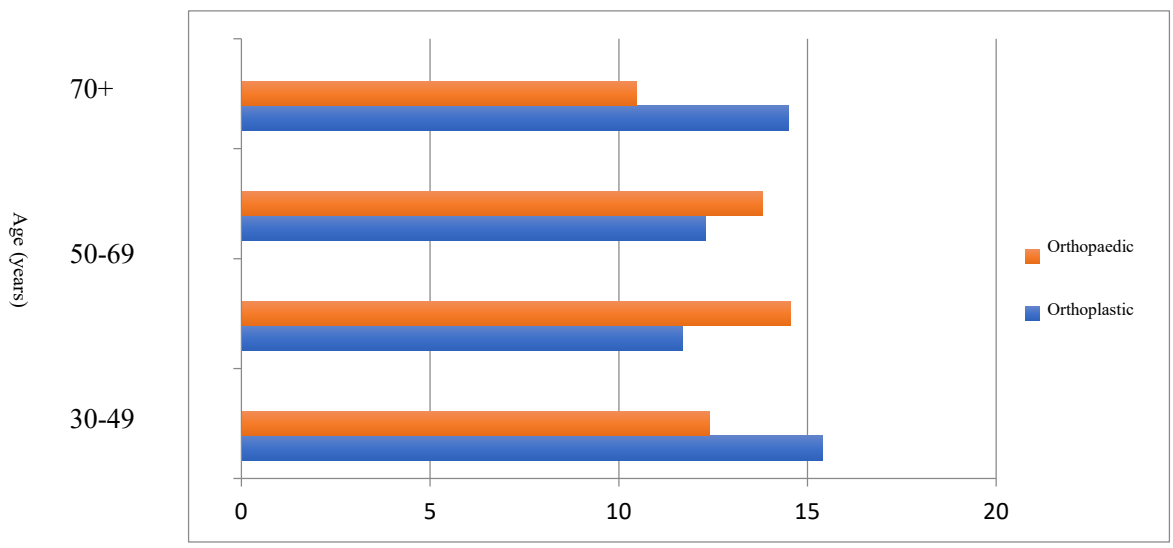

Distance $\mathrm{km}$

Figure 5. Visual representation, average distance travelled by patients of each age category 
orthoplastic group gender was split 50:50, males represented $81.5 \%$ of the patients in the orthopaedic group. The average distance travelled by all females was $11.4 \mathrm{~km}$ and the average distance travelled by all males was $14.9 \mathrm{~km}$ (Figure 6).

In the orthoplastic group the mean distance was less obviously split by gender.

\section{Age and gender comparisons}

The distribution of genders at each age was analysed. Of the orthoplastic patients the majority of the sample was made up of female patients over the age of 70, making up 33\% of the orthoplastic data set. For the orthopaedic patients $33 \%$ were young males. Figure 7 shows the spread of the whole data set according to age and gender.

For the orthoplastic patients: the males under 30 travelled the furthest distance. For orthopaedic patients the furthest distance was travelled by males over the age of 70 .

\section{Distance to Salford}

The average distance from all patients' homes to Salford Royal was $16 \mathrm{~km}$ with a range of $31.8 \mathrm{~km}$. the shortest distance is $4.5 \mathrm{~km}$ and the largest $36.3 \mathrm{~km}$. For 20 patients there would be a decrease in journey time, this represents $35 \%$ of the population studied. The largest increase in journey was $+11.1 \mathrm{~km}$ and the largest decrease in journey distance was $-11.1 \mathrm{~km}$. There was an average increase in distance for all patients of $1.6 \mathrm{~km}$. The distribution of distances of patients houses from UHSM and Salford can be seen is table 4. More patients would travel further distances to Salford, with $86 \%$ of patient's houses being more than $10 \mathrm{~km}$ away, compared to $60 \%$ of patients houses being more than $10 \mathrm{~km}$ away from UHSM.

The trend of distances from patients homes to UHSM and Salford showed an proportional relationship, so that those who had a longer journey time than average to UHSM would also have a longer than average journey time to Salford, this relationship is shown in figure 8.

\section{Transfer to Salford}

For orthoplastic patients who require both orthopaedic and plastics specialties unavailable at Salford there would be additional transport distances. The average distance from patients homes to Salford and then transfer distance from Salford to UHSM was $25.4 \mathrm{~km}$ an increase of $57 \%$. Figure 9 compares this total distance including transfer to the previous home to hospital average and Salford to home averages.

\section{Discussion}

The patients in my report travelled on average $1.1 \mathrm{~km}$ further to their point of care than patients in a national study [7]. This value was after $5 \%$ of the data was removed due to being outliers, which would have further increased the average distance, travelled by patients. This report gives an insight to the geographical area served by the trauma

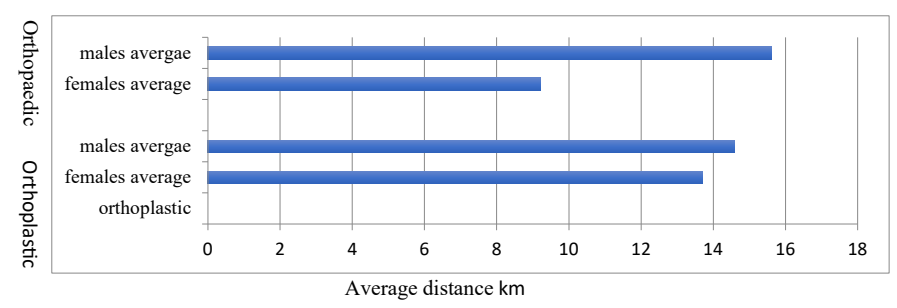

Figure 6. Average distance travelled by gender

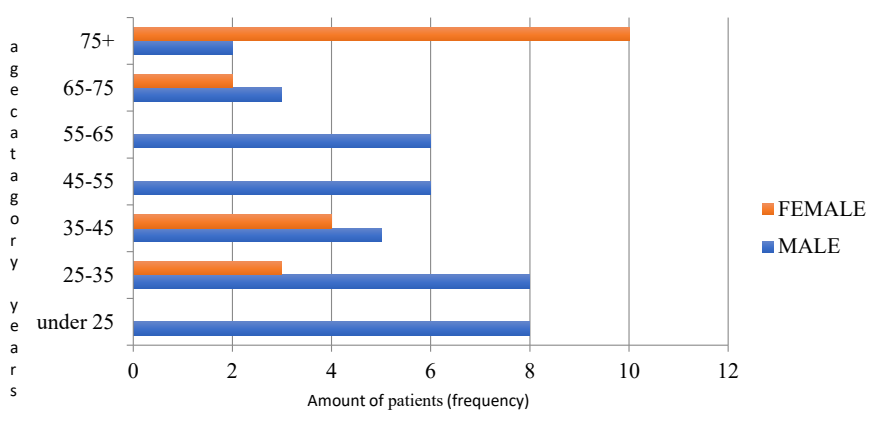

Figure 7. Bar chart showing gender distribution of each age group

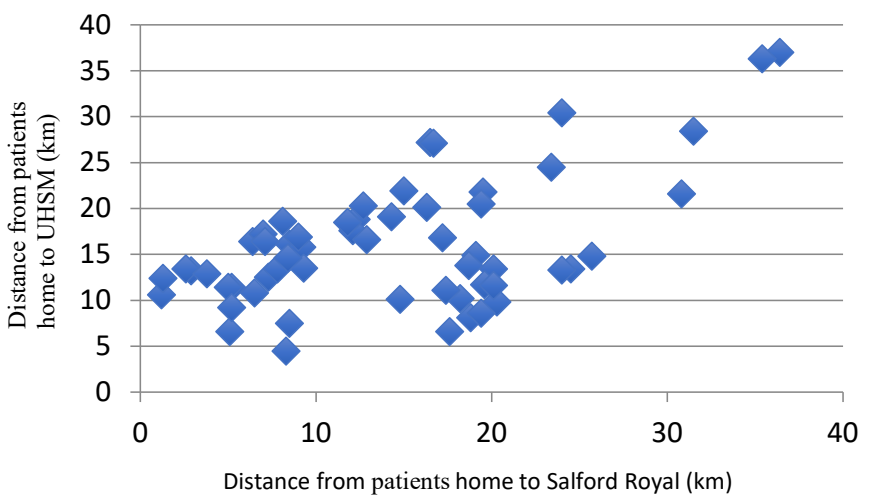

Figure 8. Graph showing the trends in data based on distances from patients homes to UHSM and Salford Royal

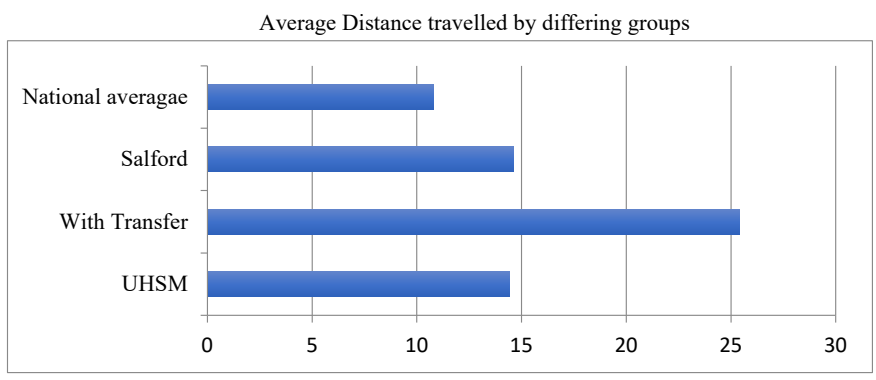

Figure 9. Representing the differences between patient to home distances for the orthoplastic patients compared to distance including transfer and the national average distance travelled to access trauma care

Table 2. Table showing differences in distances of patients' houses to UHSM and Salford

\begin{tabular}{|c|c|c|c|c|c|c|}
\hline \multicolumn{2}{|c|}{ Distance category } & $\mathbf{0 - 5} \mathbf{~ k m}$ & $\mathbf{5 - 1 0} \mathbf{~ k m}$ & $\mathbf{1 0 - 1 5} \mathbf{~ k m}$ & $\mathbf{1 5 - 2 0} \mathbf{k m}$ & $\mathbf{2 0 +} \mathbf{k m}$ \\
\hline \multirow{2}{*}{$\begin{array}{c}\text { Number of } \\
\text { patients }\end{array}$} & UHSM & 5 & 18 & 7 & 15 & 12 \\
\cline { 2 - 7 } & Salford & 1 & 7 & 23 & 13 & 13 \\
\hline
\end{tabular}

services at UHSM, with a cluster of patients' homes being to the East of UHSM.

There was an average distance of $14.4 \mathrm{~km}$ between patients' homes and UHSM, which is not hugely greater than regular distances travelled for other purposes such as commuting with a UK average distance of $14 \mathrm{~km}$ a journey that would be made usually on a daily basis. There have been attempts by many trusts to combine the purposes of visits, so that patients have to make fewer journeys for rehabilitation and outpatient clinics $[2,7]$. One cannot assume that family and friends visiting inpatients after trauma would be coming from the same areas, meaning that although the distance travelled by the patients from their homes to UHSM in the study was further than the national average it is not an unreasonable distance. 
The age and gender comparisons in my study saw that open fractures of the lower limb were more common in males under 30 and females over the age of 70 . This coincides with the findings of other studies that the mechanism of injury is shifting from RTAs to small height falls, making trauma injuries more common in the elderly than previously. The elderly female cohort are more likely to have slower recovery rates and poor responses to rehabilitation, they are also more likely to be immobile and require public transport tot hospital. This will mean distances travelled by this population if lengthened would have the largest impact. The data sets were uneven in this representation with many more elderly females needing orthoplastic care than not.

There were no consistent patterns between distance travelled and patient age overall, I would have expected that elderly patients travelled shorter distances based on theories of mechanism of injury. Small height falls are likely to occur at home, so I would have expected patients to be taken to the nearest hospital equipped for trauma care. I would have expected younger patients most commonly injured by RTAs to have a larger distance from home to hospital as the injuries could occur on any road link independent of their home addresses. This pattern was not seen, possibly due to the small sample size used in this study. It was also not possible to compare the mechanism of injury or map the site where the injury occurred, which could have exposed these trends. This pattern was shown slightly in the orthoplastic group with young males travelling the furthest for treatment however it did not show linear progression through the age categories.

If Salford were to become the single site patients in this study would have to travel an average of $1.6 \mathrm{~km}$ more, an average distance of $16 \mathrm{~km}$. Only $35 \%$ of patients benefit from a shorter distance. There are disadvantages of further travel such as meeting criteria for antibiotic administration and debridement according to BOAST4 criteria. If adherence to these criteria is hindered it would suggest increasing distances to hospital would not adhere to best evidence principles. Furthering travel distances for outpatients could present the need to restructure outpatient care.

When considering transfer distances for patients needing both orthopaedic and plastic surgical intervention, which is not available at Salford, the distances travelled by patients more than doubled. This is an excessive feat for patients who have sustained such substantial injuries, the risks involved with patient transfer are increased in trauma scenarios.

\section{Future directions}

It would be beneficial to consider repeating this audit with a larger study population, so that analysis would be more representative of the whole population. This audit could be added to by using journey times of patients, as this would allow the true impact of a changing site to be appreciated in terms of access. Questionnaires for patients who use the outpatient and inpatient services would be a useful addition; this would help establish modes of transport to and from the hospital and the impact of changing site on a social and economic level.

Collecting data about the site of injury and mechanism of injury would allow analysis of distance or time to the patients first point of care would also be beneficial in accessing access to trauma care.

Costing studies into the economic cost of moving plastic surgical facilities to Salford or the transfer or very unwell patients would allow for the feasibility of the recommendations to be assessed in another light.

\section{Conclusions}

The patients treated at UHSM between May 2013 and May 2015 for open fractures of the lower extremity had longer home to hospital distances than the national average. Suggesting they travelled further to access trauma care.

Open fractures of the lower extremity were more common in young males and elderly females adding to previous conclusions made by the literature.

There was no link between age or gender and the distances from home to hospital. This was unexpected and may possibly be due to the limitations of using a relatively small data set. Repeating this audit with a larger data set would allow more reliable analysis.

No definitive conclusions about distance to point of care can be drawn using home to hospital distances. This hinders the ability to draw conclusions about the impacts of moving to one site and adherence to BOAST4 criteria in terms of timely management recommendations, however the consideration of transfer adding $11 \mathrm{~km}$ to each patient journey regardless of their starting point can lead to questioning on the application of best practice principles in these cases.

If care were moved to the suggested site, patients' home to hospital distances would increase to an average of $16 \mathrm{~km}$, this is considerably above the national average.

The transfer of critically unwell patients for plastic surgical intervention that would not be available at Salford but would be available Wythenshawe could lead to loss of continuity of care and risks not adhering to protocol.

Salford Royal could not operate as a trauma network alone as it would be unable to treat open lower limb trauma onsite; this is a requirement of a trauma centre and needs both plastic and orthopaedic surgical specialties.

The option of using 2 sites or considering the site within the Greater Manchester Collaborative with the required facilities would be favourable.

The social and ethical implications of moving services to one site should be considered before any changes enforced, patient impact questionnaires and public involvement would facilitate this.

\section{References}

1. Adult major trauma services greater Manchester. Healthier together gm 2015

2. Major trauma services-Emergency and urgent care-NHS choices. NHS 2016.

3. Wordsworth M, Lawton G, Nathwani D, Pearse M, Naique S, et al. (2016) Improving the care of patients with severe open fractures of the tibia: the effect of the introduction of major trauma networks and national guidelines. Bone Joint $J$ 98-98B: 420-424. [Crossref]

4. Nayagam S, Graham K, Pearse M, Nanchahal J (2011) Reconstructive surgery in limbs The case for the orthoplastic approach. Ann Plast Surg 66: 6-8. [Crossref]

5. McCullough AL, Haycock JC, Forward DP, Moran CG (2014) II. Major trauma networks in England. Br J Anaesth 113: 202-206. [Crossref]

6. Kehoe A, Smith JE, Edwards A, Yates D, Lecky F (2015) The changing face of major trauma in the UK. Emerg Med J 32: 911-915. [Crossref]

7. Focus On: Distance from home to emergency care. Quality watch, Nuffield trust, the health foundation 2014.

8. Travel to work: Personal travel factsheet. Department of transport, national statistics.

9. Hendrickson SA, Khan MA, Verjee LS, Rahman KM, Simmons J, et al. (2016) Plastic surgical operative workload in major trauma patients following establishment of the major trauma network in England: a retrospective cohort study. J Plast Reconstr Aesthet Surg 69: 881-887. Web. 
10. BOAST 4: The management of severe open lower limb fractures. British Orthopaedic Association and British Association of Plastic, Reconstructive and Aesthetic Surgeons. Standard for trauma 2009

11. Jansen JO, Morrison JJ, Tai NR, Midwinter MJ (2015) A survey of major trauma centre staffing in England. J R Army Med Corps 161: 341-344. [Crossref]

12. Prodromidis AD, Charalambos CP (2016) The 6-hour rule for surgical debridement of open tibial fractures. J Orthop Trauma 30: 397-402. [Crossref]

13. Sabet FP, Tabrizi KN, Khankeh HR, Saadat S, Abedi HA, et al. (2016) Road traffic accident victims' experiences of return to normal life: A qualitative study. Iran Red Crescent Med J 18: e29548.

14. Peer review visit report: GMMTCC Definitive care measures. Richard McMahon. NHS 2015.
15. National peer review report: Major trauma 2015. National peer review programme. 2015.

16. CQC inspection report: Salford Royal hospital/Wythenshawe hospital/Manchester Royal Infirmary. Care quality commission. 2015/2016/2016.

17. Salford Royal NHS foundation trust: Information about services. SRFT 2016

18. Manchester Royal Infirmary: Our services. Central Manchester university hospitals NHS foundation trust 2016

19. UHSM: Our specialist services. University hospital of south Manchester NHS foundation trust. 2016.

20. Nachahal J, Nayagam S, Khan U, Moran C, Barret S, et al. (2009) Standards for the management of Open Fractures of the lower limb.

Copyright: $\odot 2018$ Hawthorne G. This is an open-access article distributed under the terms of the Creative Commons Attribution License, which permits unrestricted use, distribution, and reproduction in any medium, provided the original author and source are credited. 